\title{
Electrochromic Composite Films of Tunable Color based on Polyoxometalate and Auxiliary Materials
}

\author{
Shuping LIU \\ College of Tourism and Cuisine, Harbin University of Commerce, Harbin 150076, China \\ email: liusp201@163.com
}

Keywords: Electrochromic; Tunable color; Polyoxometalate; Layer-by-Layer

\begin{abstract}
The addition of another electrochromic component has been considered to be an important way to obtain multicolor film. Herein, we fabricated the composite films containing polyoxometalate (POM) clusters, auxiliary materials and polyelectrolyte on quartz and FTO substrates by layer-by-layer assemble method (LbL). The composite film shows high optical contrast, suitable response time and low operation potential. The interesting feature of the electrochromic film is its adjustable color by reduction of polyoxometalate at different potentials. The results provide valuable information for exploring application in tunable-color electrochromic devices.
\end{abstract}

\section{Introduction}

Electrochromic materials that change their optical properties during electrochemical oxidation and reduction have displayed great potential for applications such as smart windows, antiglare mirrors and displays [1,2]. Recently, much effort has been dedicated to the improvement of the electrochromic properties including optical contrast, coloration efficiency and multicolor. However, there are only a few examples of materials that report on having multicolor electrochromic properties. Polyoxometalates (POMs), a well-known class of transition metal oxide nanoclusters with intriguing structures and diverse properties, have attracted increasing attention $[3,4]$. However, in comparison with a larger number of researches on other electrochromic compounds, such as transition metal oxides, Prussian blue, viologens and conductive polymers, the study of electrochromism of POMs has been rarely reported.

In recent years, the layer-by-layer (LbL) method relied on alternately electrostatic adsorption of oppositely charged species has been widely utilized to fabricated composite films [5.]. There were many reports the POMs immobilized in the composite films by the LbL method [6,7]. Recently, Xu et al. prepared two composite films based on POMs, $\left[\mathrm{P}_{2} \mathrm{~W}_{17} / \mathrm{Cu}(\mathrm{phen})_{2}\right]_{30}$ and $\left[\mathrm{P}_{2} \mathrm{~W}_{17} / \mathrm{Fe} \text { (phen) }\right]_{30}$ (phen $=1,10$-phenithroline) [8]. These films displayed tunable colors by applying different potentials, suggesting that more auxiliary materials should be incorporated into the films to construct multicolor materials.

In this paper, we incorporated auxiliary materials (metryl orange or neutral red) and POM composite film by the LbL method. The composite film demonstrates tunable color depending on the extent of the reduction of POM with different applied potentials. Furthermore, the performance of high optical contrast, suitable response time, low operation potential and tunable color should be promising to meet the requirement for developing fexible electrochromic devices.

\section{Experimental details}

POM was prepared according to the literature method and identified by UV-vis absorption spectra. Poly(styrenesulfonate) (PSS),poly(ethylene imine) (PEI), metryl orange (MO) and neutral red (NR) were of analytical grade and used without further purification. F-doped $\mathrm{SnO}_{2}$ transparent conducting glass substrates (FTO) were purchased from Yingkou OPV Tech New Energy Co., Ltd $\left(7-8 \Omega \square^{-1}\right)$.

Layer-by-layer films were assembled onto FTO-coated glass and quartz substrates. First, the 
substrates were immersed into PEI solution $\left(1 \times 10^{-4} \mathrm{M}\right)$ for $2 \mathrm{~h}$. Then the PEI-supported substrates were alternately dipped into the PSS $\left(5 \times 10^{-3} \mathrm{M}\right)$, PEI $\left(5 \times 10^{-3} \mathrm{M}\right)$, POM $\left(1 \times 10^{-2} \mathrm{M}\right)$ and metryl orange $\left(1 \times 10^{-3} \mathrm{M}\right)$ solution for $10 \mathrm{~min}$. After deposition of each layer, the substrates were rinsed with deionized water after each dipping. Repeat the above process until the desired number of composite film (referred to as POM-MO) was obtained. For comparison, POM-NR film was also prepared on an FTO substrate by the same method.

Scanning electron micrographs (SEM) were obtained with a Hitachi S-4800 instrument. UV-vis absorption spectra were recorded on Varian Cary 50 Conc UV-vis spectrophotometer. All the electrochemical experiments were performed on the CHI 605C electrochemical workstation (Shanghai Chenhua Instrument Factory, China). The electrolyte was $1 \times 10^{-4} \mathrm{M} \mathrm{NaAc}-\mathrm{HAc}(\mathrm{pH}=$ 3.5).

\section{Results and discussion}

The composite film was assembled onto FTO-coated glass via ionic attraction of oppositely charged POM, MO, PSS and PEI (see the inset in Fig. 1). UV-vis spectroscopy was used to monitor the layer-by-layer assembling process of POM-MO composite films owing to its facility and usefulness in evaluating the growth process of the layers. Fig. 1 shows UV-vis transmittance spectra of POM-MO (number of layer: 1-6) assembled on a quartz substrate. The film displays transmittance peak at $280 \mathrm{~nm}$, which results from an overlap of POM (282 nm) and MO (272 nm). The result suggested that the POM, MO and PSS and PEI were constantly deposited in the composite film.

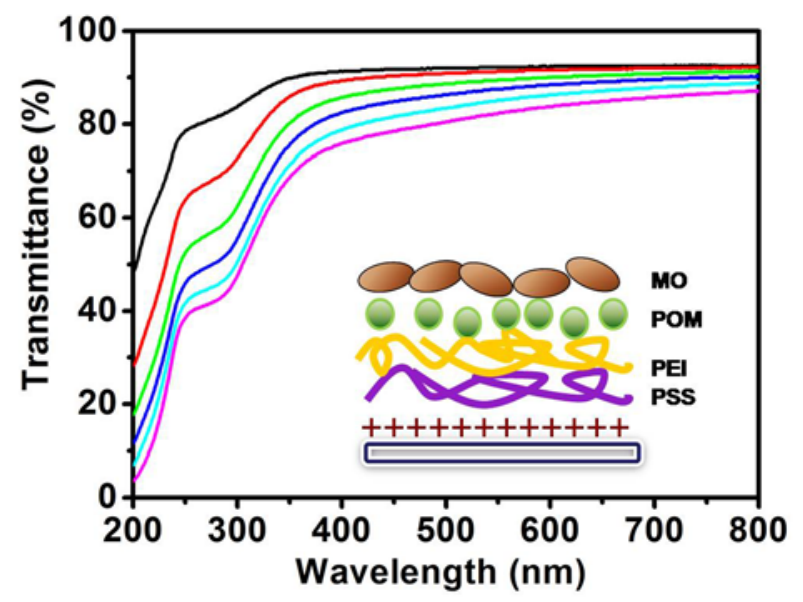

Fig. 1 UV-vis transmittance spectra of POM-MO film (number of layer: 1-6). Inset: schematics of self-assembly of a POM-MO film.
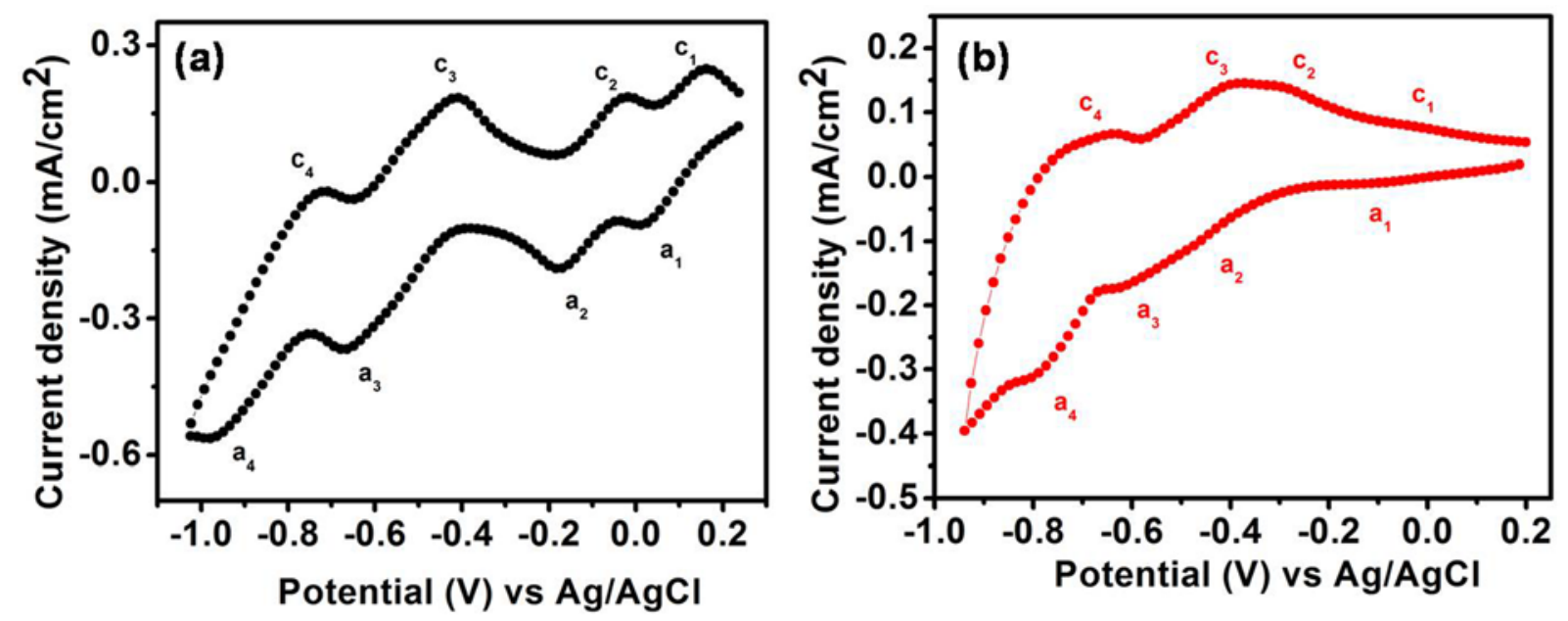

Fig. 2 (a) CV of POM solution at a scan rate of $50 \mathrm{mV} / \mathrm{s}$. (b) POM-MO composite film in $1 \times 10^{-4} \mathrm{M}$ NaAc-HAc $(\mathrm{pH}=3.5)$ solution. 
Cyclic voltammograms (CV) was used to monitor the electrochemical behavior of the films. Fig. 2a shows the $\mathrm{CV}$ of the POM solution using a glassy carbon as the working electrode with a scan rate of $50 \mathrm{mV} / \mathrm{s}$. four pairs of reversible redox peaks $\left(c_{1} / a_{1}, c_{2} / a_{2}, c_{3} / a_{3}\right.$ and $\left.c_{4} / a_{4}\right)$ are observed, which correspond to four recersible (one-, one-, two- and two-electron) reduction processes. Fig. $2 b$ displays the CV of the as-prepared POM-MO film. The film exhibit four couples of redox waves, which is similar to the CV curve of the POM solution.

The electrochromic properties of the POM-MO film were investigated by double potential experiments as well as absorption measurements. The optical contrast is probably the most important parameter in evaluating electrochromic materials. Fig. 3 displays the current and transmittance of the POM-MO film at $650 \mathrm{~nm}$ under a square wave potential of $-1.0 \mathrm{~V}$ and $+1.0 \mathrm{~V}$. As shown in Fig. 3, the optical contrast of the composite film is $31.0 \%$. Then response time is defined as the requirement for $90 \%$ of the total absorbance change to be reached. The switching times were $2.4 \mathrm{~s}$ for coloration and $3.1 \mathrm{~s}$ for bleaching.

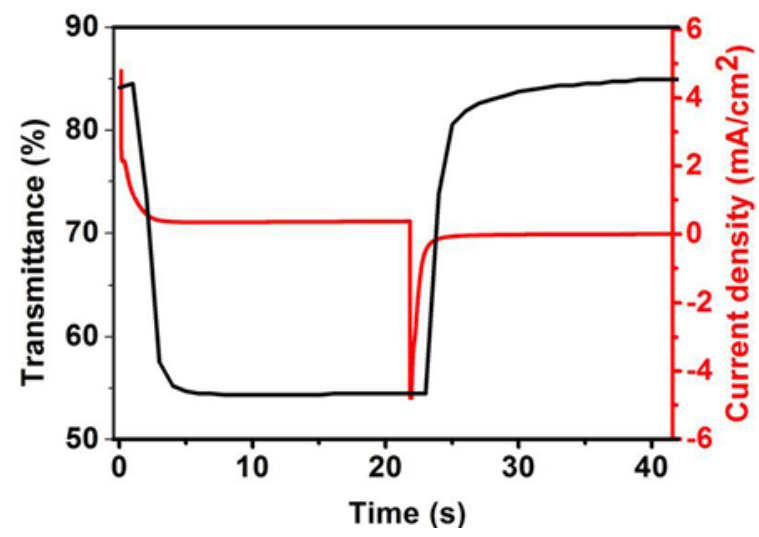

Fig. 3 chronoamperometry curves (red line) and optical transmittance change (black line) at $650 \mathrm{~nm}$ of POM-MO film during subsequent double-potential steps $(-1.0 \mathrm{~V}$ and $+1.0 \mathrm{~V})$.

Fig. 4 displays visible spectra of POM-MO and POM-NR composite films under different potentials. As shown in Fig. 4a, with decreasing potential, the transmittance decreases gradually, the coloration degree deepens and the maximum transmittance peak changes. Upon electrochemical reduction, POM exhibits blue color, meanwhile, a broad transmittance band with a maximum at approximately $650 \mathrm{~nm}$ is observed. When more negative potentials are applied, the POM is reduced to different extent of blue colors, mixed with yellow of MO, to display yellow, light blue and dark blue, respectively.
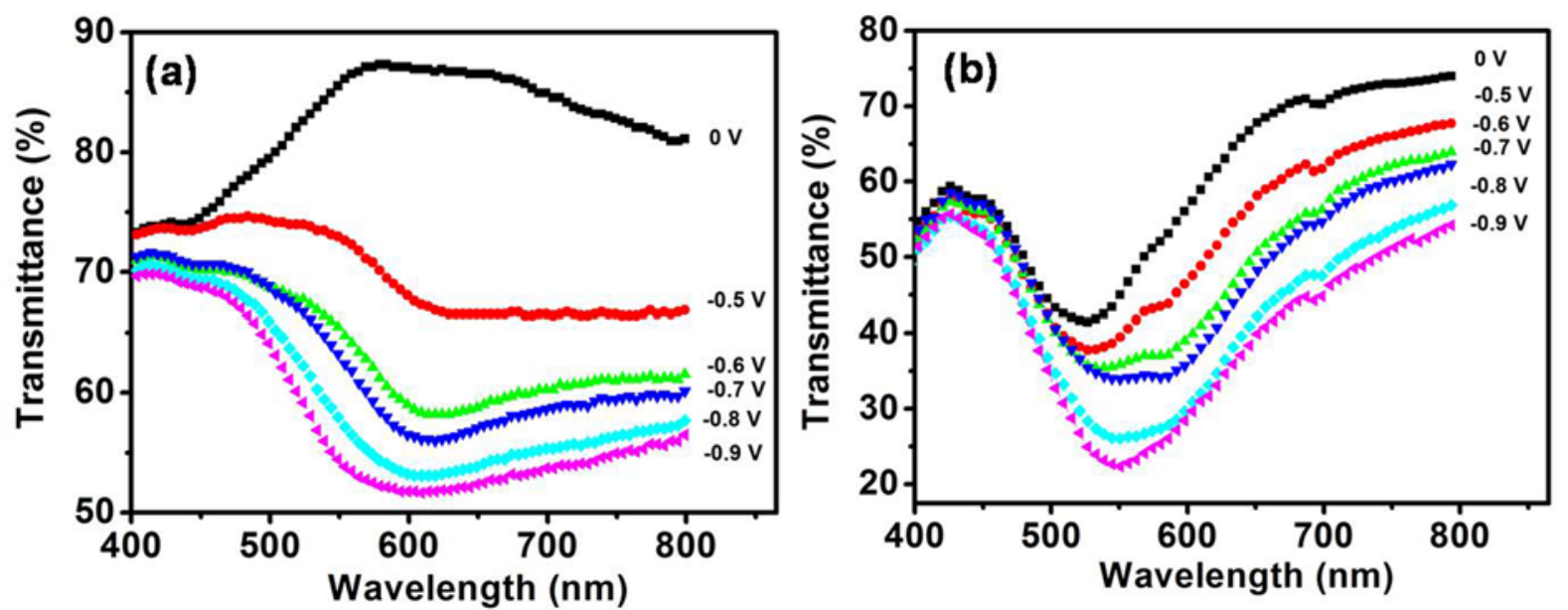

Fig. 4 Visible spectra of POM-MO (a) and POM-NR (b) films under different potentials (From top to bottom: $0 \mathrm{~V},-0.5 \mathrm{~V},-0.6 \mathrm{~V},-0.7 \mathrm{~V},-0.8 \mathrm{~V}$ and $-0.9 \mathrm{~V})$. 
In comparison, the NR displays deep pink without applying potential for the POM-NR film (Fig. 4b). When more negative potentials are applied, the POM is reduced to different extent of blue color, mixed with deep pink color of NR cations, to show deep pink, light purple, dark purple-blue, respectively.

\section{Conclusion}

In conclusion, the nanocomposite material composed of POM, MO, PEI and PSS was successfully fabricated using LbL assembly method. The composite film is found to switch from yellow to dark blue, because POM is reduced to different extent blue colors, mixed with yellow of MO. In addition, the composite film displays high electrochromic properties with high optical contrast and suitable response time. Therefore, the composite film based on POM and MO with multicolor electrochromic properties could become a promising candidate for possible applications in electrochromic devices.

\section{Acknowledgement}

This work was financially supported by the Natural Science Foundation of China (Grant No. 21301041 and No. 31201376) and Harbin University of Commerce Doctor Start-up Fund Research (No. 12DW030).

\section{References}

[1] Nguyen Minh Vuong, Dojin Kim, Hyojin Kim. Electrochromic properties of porous $\mathrm{WO}_{3}-\mathrm{TiO}_{2}$ core-shell nanowires [J]. Journal of Materials Chemistry C. 2013 (1) 3399-3407.

[2] Feng Lin, Jifang Cheng, Chaiwat Engtrakul, Anne C. Dillon, Dennis Nordlund, Rob G. Moore, Tsu-Chien Weng, S. K. R. Willians, Ryan M. Richards. In situ crystallization of high performing $\mathrm{WO}_{3}$-based electrochromic materials and the importance for durability and switching kinctics [J]. Journal of Materials Chemistry. 2012 (22) 16817-16823.

[3] Shuping Liu, Lin $\mathrm{Xu}$, Fengyan $\mathrm{Li}$, Bingbing $\mathrm{Xu}$, Zhixia Sun. Enhanced electrochromic performance of composite films by combination of polyoxometalate with poly(3,4-ethylenedioxythiophene) [J]. Journal of Materials Chemistry. 2011 (21) 1946-1952.

[4] Shuping Liu, Lin Xu, Fengyan Li, Weihua Guo, Yan Xing, Zhixia Sun. Carbon nanotubes-assisted polyoxometalate nanocomposite film with enhanced electrochromic performanc[J]. Electrochimica Acta. 2011 (56) 8156-8162.

[5] Jinlin Lu, Wanshuang Liu, Han Ling, Junhua Kong, Guoqiang Ding, Dan Zhou and Xuehong Lu. Layer-by-layer assembled sulfonated-graphene/polyaniline nanocomposite films: enhanced electrical and ionic conductivities, and electrochromic properties[J]. RSC Advances. 2012 (2) 10537-10543.

[6] Di Zhang, Yeqiong Zhang, Huiyuan Ma, Hong Yan, Yongbin Song. Fabrication of a 12-tungstophosphate and cadmium oxide composite film and its properties[J]. Materials Chemistry and Physics. 2014 (144) 369-376.

[7] Shuping Liu, Lin Xu, Guanggang Guo, Bingbing Xu, Weihua Guo. Electrochromic multilayer films with enhanced stability based on polyoxometalate and $\mathrm{TiO}_{2}[\mathrm{~J}]$. Materials Chemistry and Physics. 2009 (116) 88-93.

[8] Guanggang Gao, Lin Xu, Wenju Wang, Wenjia An, Yunfeng Qiu, Zhengqing Wang, Enbo Wang. Electrochromic Multilayer Films of Tunable Color by Combination of Copper or Iron[J]. The Journal of Physical Chemistry B. 2005 (109) 8948-8953. 